\title{
Amino Acid-Based Metabolic Indexes Identify Patients With Chronic Obstructive Pulmonary Disease And Further Discriminates Patients In Advanced BODE Stages
}

This article was published in the following Dove Press journal:

International Journal of Chronic Obstructive Pulmonary Disease

\author{
Wei-Ke Kuo (iD) \\ Yu-Chih Liu ${ }^{1,2}$ \\ Chien-Ming Chu' \\ Chung-Ching Hua $\left(\mathbb{D}^{1,2}\right.$ \\ Chih-Yu Huang $\mathbb{D}^{\prime}$ \\ Min-Hui Liu $\mathbb{D}^{2,3}$ \\ Chao-Hung Wang $\mathbb{D}^{2,3}$ \\ 'Division of Pulmonary, Critical Care, and \\ Sleep Medicine, Department of Internal \\ Medicine, Chang Gung Memorial \\ Hospital, Keelung, Taiwan; ${ }^{2}$ Chang Gung \\ University, College of Medicine, Taoyuan, \\ Taiwan; ${ }^{3}$ Heart Failure Research Center, \\ Division of Cardiology, Department of \\ Internal Medicine, Chang Gung Memorial \\ Hospital, Keelung, Taiwan
}

Background: The BODE index is a multidimensional grading system for predicting the prognoses of patients with chronic obstructive pulmonary disease (COPD). This study investigated whether an amino acids-based metabolic profile developed for heart failure patients (including histidine, ornithine, phenylalanine, and leucine) could identify COPD patients and further discriminates COPD patients in advanced BODE stages.

Methods: Ultra-performance liquid chromatography was performed on 119 participants, including 75 COPD patients at different BODE stages and 44 normal controls. Albumin, prealbumin, transferrin, high sensitivity C-reactive protein, and hand grip strength were also measured. Receiver operating characteristic curves and area under curves were used for estimation.

Results: The BODE points in our patients were 3.29 [95\% confidence interval $(\mathrm{CI})=2.74-3.85$ ] Compared to normal controls, COPD patients had lower leucine but higher ornithine levels. A COPD score, developed based on leucine and ornithine, significantly discriminated COPD from normal controls [odds ratio $(\mathrm{OR})=2.71,95 \% \mathrm{CI}=1.83-4.04, \mathrm{p}<0.001$ ]. A COPD score of $\geq 3.00$ had an OR of $15.58(95 \% \mathrm{CI}=5.96-40.73, \mathrm{p}<0.001)$. In COPD patients from BODE 1 to BODE 4, the levels of histidine, ornithine and phenylalanine increased significantly. In multivariable analysis, histidine and phenylalanine were independently able to distinguish BODE stages 3 and 4 from BODE 1 and were adopted to develop a metabolic score. Metabolic scores identified patients at BODE 3 and $4(\mathrm{OR}=2.74,95 \% \mathrm{CI}=1.41-5.29, \mathrm{p}=0.003)$ better than hand grip strength, high sensitive C-reactive protein, albumin, pre-albumin, and transferrin value. A metabolic score of $\geq 9.53$ significantly discriminated $\mathrm{BODE} 3$ and 4 from $\mathrm{BODE} 1$ and $2(\mathrm{OR}=8.56,95 \% \mathrm{CI}=2.77$ 26.39, p <0.001).

Conclusion: Amino acid-based COPD score and metabolic score discriminate COPD patients from normal controls, and identify patients in advanced stages of COPD.

Keywords: chronic obstructive pulmonary disease, BODE, metabolomics, amino acids, heart failure

\section{Introduction}

Chronic obstructive lung disease (COPD), currently the third leading cause of mortality worldwide, results in a great social and economic burden. ${ }^{1}$ This common and preventable disease is characterized by persistent and progressive airflow limitation associated with inflammation caused by exposure to noxious particles, especially tobacco. ${ }^{2}$ The BODE index, a multidimensional grading system, comprises body
Heart Failure Research Center, Divis of Cardiology, Department of Internal Medicine, Chang Gung Memorial Hospital, No. 222, Maijin Road, Anle District, Keelung City 204, Taiwan

Tel +886-2-2431313I ext 2245

Fax +886-2-24335342

Email bearty54@gmail.com 
mass index (BMI), forced expiratory volume in one second (FEV1), dyspnea score (modified Medical Research Council questionnaire (mMRC)), and exercise capacity (six-minute walking distance (6MWD)). ${ }^{3}$ Studies show that a single BODE index measurement can predict morality and hospital admission for COPD. ${ }^{3-5}$ However, the BODE index requires use of physiological tests and questionnaires, which are somewhat subjective and not easily reproducible, which may limit the reliability of the BODE index for clinical use. ${ }^{6}$ There is an unmet need to identify objective biomarkers for assessing COPD severity.

Metabolomics is a comprehensive tool for investigating the biochemical changes in cells, tissue, and body fluids with the potential to represent an individual's health status and guide disease treatment. It is a rapidly growing field ${ }^{7}$ and several metabolomics studies have documented different metabolism patterns between COPD patients and normal controls. ${ }^{8-11}$ However, the results of such research have not yet been applied for clinical use, probably due to the limited availability of mass spectrometry and the complexity of multi-metabolite measurement. ${ }^{12,13}$ Previously, we proved the diagnostic and prognostic value of metabolomics in plasma for heart failure (HF) patients. ${ }^{14}$ Next, we created a simplified metabolic panel by measuring only histidine, ornithine, phenylalanine and leucine utilizing ultra-performance liquid chromatography (UPLC). ${ }^{12,15}$ This profile provided not only diagnostic and prognostic value during both the acute stage and outpatient phases, but also indicated muscle breakdown and functional status. ${ }^{12,15}$ COPD and HF share similar symptoms, signs, pathogenic mechanisms, skeletal muscle alterations, as well as similar exercise intolerance caused by cachexia. ${ }^{16,17}$ We decided to test whether the amino acidbased metabolic profile we developed for HF could be applied to COPD patients.

Thus, the aims of this study were: 1) to investigate the relationship of four amino acids (histidine, ornithine, phenylalanine, and leucine) to the presence of COPD and to the BODE index; 2) to investigate the ability of this amino acid-based panel to discriminate COPD patients from normal controls and discriminate between different BODE stages compared to traditional assessment tools, such as parameters for nutrition (albumin, pre-albumin, transferrin), inflammation (C-reactive protein), and muscular function (hand grip strength); and 3) to see whether a metabolic panel derived from HF patients could be also applied to COPD patients.

\section{Methods}

\section{Patients And Study Design}

From May 2017 to September 2018, patients with all severity levels of airflow constriction were consecutively enrolled if they were between 40 and 90 years old and fulfilled the criteria for COPD according to the Global Initiative for Chronic Obstructive Lung Disease (GOLD) guidelines. ${ }^{2}$ Exclusion criteria included: patients who were bed-ridden for more than three months and/or unable to stand alone; patients with a serum creatinine of $>3 \mathrm{mg} / \mathrm{dl}$; patients with active cancer; and patients with disorders that might compromise their survival within the next six months. Normal controls were ages 20 to 85 years, had no significant systemic disease, and were not on any medications. Written informed consent was obtained from all patients. The study was designed and carried out in accordance with the principles of the Declaration of Helsinki and with approval from the Ethics Review Board of Chang Gung Memorial Hospital (IRB/CGMH, No.201700159B0C102).

\section{Clinical Variables Related To COPD}

The following variables were evaluated in COPD patients: age; sex; comorbidities; FEV1 $;{ }^{18}$ mMRC; ${ }^{19}$ chronic obstructive lung disease assessment test (CAT); ${ }^{20} 6 \mathrm{MWD} ;{ }^{21}$ oxygen saturation ( $\mathrm{SpO} 2)$; left ventricular ejection fraction (LVEF); GOLD stage, ${ }^{2}$ COPD medication (inhaler and oral corticosteroids); hand grip strength of the dominant hand; ${ }^{22}$ and BODE index (BODE stages 1, 2, 3 and 4 were defined by BODE $0-2,3-4,5-6$, and $7-10$ points, respectively). ${ }^{3}$

\section{Blood Sampling And Examination}

To measure met abolites, we collected blood samples of COPD patients in EDTA-containing tubes in the early morning after overnight fasting. Plasma was analyzed by metabolomics workflow. For normal controls, we collected blood samples for metabolites at enrollment. Measurement of other parameters, including high sensitive C-reactive protein (hsCRP), albumin, pre-albumin, transferrin, and fasting blood sugar, was conducted in the central core laboratory.

\section{Ultra-Performance Liquid Chromatography (UPLC)-Based Measurement}

The plasma samples $(100 \mu \mathrm{L})$ were precipitated by $10 \%$ sulfosalicylic acid. After protein precipitation and centrifugation, we initiated derivatization using $\mathrm{AQC}$ in acetonitrile. 
Next, amino acids were analyzed using the ACQUITY UPLC System which consisted of a binary solvent manager, a sample manager, and a tunable UV detector. The system was controlled and data collected using Empower ${ }^{\mathrm{TM}} 2$ Software. Separations were performed on a $2.1 \times 100 \mathrm{~mm}$ ACQUITY BEH C18 column at a flow rate of $0.70 \mathrm{~mL} / \mathrm{min}$. The average intra-assay coefficient of variation was $4.3 \%$ for histidine, $4.6 \%$ for ornithine, $4.5 \%$ for leucine, and $4.6 \%$ for phenylalanine. The total coefficient of variation was $3.1 \%$ for histidine, $3.6 \%$ for ornithine, $4.1 \%$ for leucine, and 3.7\% for phenylalanine. The detection limit was $0.5 \mu \mathrm{M}$ for histidine, $2.0 \mu \mathrm{M}$ for ornithine, $0.9 \mu \mathrm{M}$ for leucine, and $3.3 \mu \mathrm{M}$ for phenylalanine. The linear range was $25-500 \mu \mathrm{M}$ for these four amino acids.

\section{Metabolite Concentration Corrected By Leucine Levels}

Previously, we demonstrated that the concentration of leucine correlated significantly with the concentration of total essential amino acids, indicating nutritional status. ${ }^{12}$ To avoid the impact of low total essential amino acid levels on the interpretation of individual amino acid concentrations, we used leucine for correction of histidine, ornithine and phenylalanine as follows. If the concentration of leucine was $\leq[$ (mean leucine level in normal subjects) - (standard deviation of leucine levels in the blood of normal subjects)], other amino acid concentrations were corrected as follows. Corrected histidine level $(\mathrm{Hc})=$ histidine level $\times[$ (mean leucine level in normal subjects) - (standard deviation of leucine levels in the blood of normal subjects)]/leucine level. If leucine level $>$ [(mean leucine level in the blood of normal subjects) - (standard deviation of leucine levels in the blood of normal subjects)], Hc = histidine level. We calculated corrected ornithine levels (Oc) and corrected phenylalanine levels (Pc) using the same equation.

\section{Statistical Analysis}

We analyzed data using SPSS 22.0 (SPSS Software, Chicago, IL, USA). Results are expressed as mean \pm standard deviation for continuous variables and number (percentages) for categorical variables. Data were compared by ANOVA and chi-square when appropriate. We tested the linear trend of the distribution of demographic and laboratory data across study groups using CochranArmitage chi-square analysis for categorical variables and linear contrast in the general linear model for continuous variables. Univariate analysis detected the associations between each variable and the study groups. Each variable that was statistically significant in the univariate analysis $(p<0.05)$ was entered into a multivariate forward stepwise logistic regression to identify independent variables capable of discriminating COPD patients from normal controls and discriminating between BODE stage 1 and BODE stages 3 and 4 . In addition, we developed a COPD score and a metabolic score using the $\beta$ estimates based on the multivariable logistic regression. Alpha level was set at 0.05 and an alpha level of 0.10 was used as the cutoff for variable removal in the automated model selection for multivariable logistic regression. To investigate the usefulness of each variable for discriminating COPD patients from normal controls and discriminating BODE stages 3 and 4 from BODE stage 1, we constructed receiver operating characteristic (ROC) curves and area under curves (AUCs). Cutoff values for the variables were identified using Youden's index. A $p$ value of $<0.05$ was considered significant.

\section{Results}

\section{Baseline Characteristics}

A total of 119 participants, including 44 normal controls and 75 COPD patients with different BODE stages values were enrolled. The data of metabolites in the normal controls were used in further analyses and to correct amino-acid concentrations. Baseline characteristics and laboratory data are shown in Tables 1 and 2. Compared to normal controls, the patients with COPD were older, and had higher hsCRP and fasting blood sugar levels. From BODE stage 1 to BODE stage 4, patients showed a significant trend toward lower BMI, FEV1, 6MWD, SpO2, along with higher mMRC points, CAT points, BODE points, along with greater percentages of patients with GOLD group D and patients receiving triple therapy. In parameters indicating nutrition, inflammation and muscular function, pre-albumin levels and hand grip strength decreased significantly from BODE stage 1 to BODE stage 4 (Table 2). However, there were no significant differences in hsCRP, albumin, and transferrin.

\section{Amino Acid-Based Profile Between COPD And Normal Controls}

To test the ability of metabolites in discriminating patients with COPD from normal controls, univariate analysis showed that COPD patients had significantly lower leucine levels, but higher Hc and Oc levels compared to normal 
Table I Demographic Data In Normal Controls And Patients With Chronic Obstructive Pulmonary Disease At Different BODE Stages

\begin{tabular}{|c|c|c|c|c|c|c|}
\hline & Normal & BODE I & BODE 2 & BODE 3 & BODE 4 & \multirow[t]{2}{*}{$p$ For Trend* } \\
\hline & $n=44$ & $n=30$ & $n=23$ & $n=14$ & $n=8$ & \\
\hline Age (years) & $53.3 \pm 5.5$ & $71.4 \pm 8.9$ & $70.2 \pm 12.3$ & $74.0 \pm 7.7$ & $71.4 \pm 7.9$ & 0.749 \\
\hline Male (\%) & $36(81.8)$ & $26(86.7)$ & $2 I(9 \mid .3)$ & $13(92.9)$ & $7(87.5)$ & 0.712 \\
\hline \multicolumn{7}{|l|}{ Co-morbidity (\%) } \\
\hline Diabetes mellitus (\%) & $0(0)$ & $5(16.7)$ & $6(26.1)$ & $4(28.6)$ & $0(0)$ & 0.776 \\
\hline Hypertension (\%) & $0(0)$ & $13(43.3)$ & $12(52.2)$ & $9(23.1)$ & $5(62.5)$ & 0.171 \\
\hline Chronic kidney disease (\%) & $0(0)$ & $0(0)$ & $\mathrm{I}(4.3)$ & $2(14.3)$ & $0(0)$ & 0.245 \\
\hline Atrial fibrillation (\%) & $0(0)$ & $3(10)$ & $2(8.7)$ & $\mathrm{I}(7 . \mathrm{I})$ & $0(0)$ & 0.401 \\
\hline Hyperlipidemia (\%) & $0(0)$ & $4(13.3)$ & $2(8.7)$ & $3(21.4)$ & $0(0)$ & 0.726 \\
\hline $\operatorname{BMI}\left(\mathrm{Kg} / \mathrm{m}^{2}\right)$ & $25.0 \pm 3.2$ & $25.0 \pm 3.2$ & $24.4 \pm 4.8$ & $22.9 \pm 3.9$ & $19.9 \pm 2.9$ & 0.001 \\
\hline FEVI (\%) & & $65.8 \pm \mid 4.1$ & $50.1 \pm 20.8$ & $47.0 \pm 12.9$ & $31.1 \pm 9.0$ & $<0.001$ \\
\hline mMRC (Points) & & $1.1 \pm 0.6$ & $1.7 \pm 0.8$ & $2.6 \pm 0.8$ & $3.3 \pm 0.7$ & $<0.001$ \\
\hline CAT (Points) & & $7.3 \pm 3.8$ & $10.0 \pm 7.4$ & $18.2 \pm 6.4$ & $23.1 \pm 8.1$ & $<0.001$ \\
\hline 6MWD (meters) & & $417.9 \pm 86.0$ & $336.3 \pm 95.6$ & $213.5 \pm 112.9$ & $164.6 \pm 97.5$ & $<0.001$ \\
\hline $\mathrm{SpO} 2(\%)$ & & $97.3 \pm 1.3$ & $97.1 \pm 1.3$ & $94.9 \pm 2.9$ & $95.3 \pm 1.4$ & $<0.001$ \\
\hline BODE points & & $1.1 \pm 0.8$ & $3.2 \pm 0.6$ & $5.4 \pm 0.5$ & $8.1 \pm 1.3$ & $<0.001$ \\
\hline GOLD (\%) & & & & & & 0.002 \\
\hline A & & $14(46.7)$ & $4(17.4)$ & $0(0)$ & $0(0)$ & \\
\hline B & & $4(13.3)$ & $5(21.7)$ & $7(50)$ & $3(37.5)$ & \\
\hline C & & $8(26.7)$ & $7(30.4)$ & $0(0)$ & $0(0)$ & \\
\hline $\mathrm{D}$ & & $4(13.3)$ & $7(30.4)$ & $7(50)$ & $5(62.5)$ & \\
\hline COPD medication & & & & & & 0.001 \\
\hline LABA or LAMA & & $2(6.7)$ & $\mathrm{I}(4.3)$ & $0(0)$ & $0(0)$ & \\
\hline LABA+LAMA & & II(36.7) & $5(21.7)$ & $2(14.3)$ & $\mathrm{I}(\mathrm{I} 2.5)$ & \\
\hline$I C S+L A B A$ & & $8(26.7)$ & $4(17.4)$ & $0(0)$ & $0(0)$ & \\
\hline$L A B A+L A M A+I C S$ & & $9(30.0)$ & $13(56.5)$ & $12(85.7)$ & $7(87.5)$ & \\
\hline Additional oral steroid & & $2(6.6)$ & $0(0)$ & $2(14.3)$ & $2(25.0)$ & 0.093 \\
\hline
\end{tabular}

Notes: Data are presented as mean \pm standard deviation and number (\%). *Comparison among patients from BODEI to BODE4.

Abbreviations: CAD, coronary artery disease; CVA, cerebral vascular accident; BMI, body mass index; FEVI, forced expiratory volume in one second; mMRC, modified Medical Research Council dyspnea scale; CAT, chronic obstructive lung disease assessment test; 6MWD, six minutes walking distance; SpO2, oxygen saturation; GOLD, Global Initiative for Chronic Obstructive Lung Disease; COPD, chronic obstructive pulmonary disease; LABA, long-acting $\beta$ adrenoceptor agonists; LAMA, long-acting muscarinic antagonists; ICS, inhaled corticosteroid.

controls (Table 3). In multivariable analysis, leucine and Oc were two independent variables for identifying COPD, and were then used to develop a "COPD score". Based on the $\beta$ estimates from the multivariable logistic regression, the COPD score was calculated as $0.036 \times$ Oc $-0.051 \times$ leucine +6.33 .

To discriminate patients with COPD from normal controls, the COPD score had an OR of $2.71(95 \% \mathrm{CI}=1.83-$ $4.04, \mathrm{p}<0.001)$. The COPD score remained statistically significant after adjusting for age, hsCRP and fasting blood sugar $(\mathrm{OR}=2.66,95 \% \mathrm{CI}=1.35-5.23, \mathrm{p}=0.005)$. Using ROC curves to evaluate the diagnostic value of COPD score, the COPD score had an AUC of 0.856 (Figure 1). Based on Youden's index, the cutoff value for COPD score was set at 3.00. A COPD score of $\geq 3.00$ significantly distinguished COPD patients from normal controls $(\mathrm{OR}=15.58,95 \% \mathrm{CI}=$ $5.96-40.73, \mathrm{p}<0.001$ ) (the sensitivity, specificity, and accuracy were $74.7 \%, 85.1 \%, 78.2 \%$, respectively). 
Table 2 Functional, Laboratory, And Metabolite Data In Normal Controls And Patients With Chronic Obstructive Pulmonary Disease At Different BODE Stages

\begin{tabular}{|c|c|c|c|c|c|c|c|}
\hline & Normal & COPD & BODE I & BODE 2 & BODE 3 & BODE 4 & p For \\
\hline & $n=44$ & $n=75$ & $\mathbf{n}=\mathbf{3 0}$ & $n=23$ & $n=14$ & $n=8$ & Irend \\
\hline Hand grip strength of dominant hand $(\mathrm{kg})$ & & & $31.6 \pm 8.7$ & $31.4 \pm 8.5$ & $26.3 \pm 7.4$ & $25.3 \pm 5.7$ & 0.021 \\
\hline $\begin{array}{l}\text { Laboratory data } \\
\text { hsCRP (mg/L) } \\
\text { Albumin }(\mathrm{g} / \mathrm{dl}) \\
\text { Pre-Albumin }(\mathrm{mg} / \mathrm{dL}) \\
\text { Transferrin }(\mathrm{mg} / \mathrm{dL}) \\
\text { Sugar (fasting) (mg/dL) }\end{array}$ & $\begin{array}{l}1.8 \pm 2.9 \\
4.4 \pm 0.3 \\
98.9 \pm 9.2\end{array}$ & $\begin{array}{l}5.6 \pm 9.9^{\ddagger} \\
4.3 \pm 0.4 \\
106.7 \pm 26.3^{\dagger}\end{array}$ & $\begin{array}{l}3.2 \pm 6.8 \\
4.4 \pm 0.5 \\
29.7 \pm 6.4 \\
243.6 \pm 31.5 \\
107.3 \pm 31.7\end{array}$ & $\begin{array}{l}6.1 \pm 9.6 \\
4.4 \pm 0.3 \\
28.1 \pm 7.6 \\
246.1 \pm 43.4 \\
108.5 \pm 24.8\end{array}$ & $\begin{array}{l}9.2 \pm 15.0 \\
4.3 \pm 0.5 \\
28.7 \pm 7.1 \\
223.0 \pm 50.5 \\
103.6 \pm 22.3\end{array}$ & $\begin{array}{l}6.6 \pm 9.2 \\
4.2 \pm 0.4 \\
23.4 \pm 4.6 \\
229.9 \pm 43.2 \\
104.9 \pm \mid 5.2\end{array}$ & $\begin{array}{l}0.282 \\
0.221 \\
0.032 \\
0.205 \\
0.714\end{array}$ \\
\hline $\begin{array}{l}\text { Metabolites }(\mu \mathrm{M}) \\
\text { Histidine } \\
\text { Ornithine } \\
\text { Leucine } \\
\text { Phenylalanine } \\
\text { Correctedhistidine }(\mathrm{Hc}) \\
\text { Corrected ornithine }(\mathrm{Oc}) \\
\text { Corrected phenylalanine }(\mathrm{Pc})\end{array}$ & $\begin{array}{l}90.3 \pm 16.1 \\
90.6 \pm 18.3 \\
150.1 \pm 22.7 \\
68.0 \pm 12.5 \\
91.3 \pm 16.1 \\
91.7 \pm 18.5 \\
68.8 \pm 12.4\end{array}$ & $\begin{array}{l}85.6 \pm 17.8 \\
99.1 \pm 32.3 \\
121.8 \pm 25.6^{\ddagger} \\
63.7 \pm 15.3 \\
98.5 \pm 18.6^{\dagger} \\
114.5 \pm 36.5^{\ddagger} \\
73.2 \pm 14.8\end{array}$ & $\begin{array}{l}81.7 \pm 11.8 \\
92.7 \pm 31.1 \\
119.6 \pm 23.8 \\
59.0 \pm 9.78 \\
94.0 \pm 14.4 \\
106.6 \pm 35.5 \\
68.1 \pm 13.1\end{array}$ & $\begin{array}{l}85.7 \pm 21.6 \\
97.4 \pm 30.7 \\
123.2 \pm 26.1 \\
65.7 \pm 18.3 \\
97.8 \pm 21.0 \\
113.9 \pm 40.1 \\
74.6 \pm \mid 5.3\end{array}$ & $\begin{array}{l}87.7 \pm 20.1 \\
105.4 \pm 27.4 \\
126.5 \pm 31.9 \\
65.8 \pm 11.7 \\
100.9 \pm 16.8 \\
121.2 \pm 24.9 \\
76.5 \pm 12.4\end{array}$ & $\begin{array}{l}96.7 \pm 19.0 \\
116.7 \pm 45.2 \\
117.7 \pm 20.7 \\
71.4 \pm 23.9 \\
113.6 \pm 23.0 \\
134.5 \pm 42.7 \\
81.8 \pm 18.7\end{array}$ & $\begin{array}{l}0.035 \\
0.048 \\
0.939 \\
0.048 \\
0.007 \\
0.046 \\
0.018\end{array}$ \\
\hline COPD score & $2.0 \pm 1.1$ & $4.2 \pm 1.9^{\ddagger}$ & $4.1 \pm 1.8$ & $4.1 \pm 2.3$ & $4.2 \pm 1.7$ & $5.2 \pm 1.5$ & 0.158 \\
\hline Metabolic score & $8.9 \pm 1.2$ & $9.0 \pm 1.4$ & $8.5 \pm 0.9$ & $9.1 \pm 1.7$ & $9.3 \pm 1.1$ & $10.1 \pm 1.5$ & 0.002 \\
\hline Metabolic score + HGS & & & $4.4 \pm 1.3$ & $5.1 \pm 1.9$ & $5.9 \pm 1.5$ & $6.9 \pm 1.7$ & $<0.001$ \\
\hline
\end{tabular}

Notes: Data are presented as mean \pm standard deviation and number (\%). ${ }^{*}$ Comparison among patients from BODEI to BODE 4 . ${ }^{\dagger} \mathrm{p}<0.05$, ${ }^{\ddagger} \mathrm{p}<0.0 \mathrm{I}$, compared to the normal controls.

Abbreviations: COPD, chronic obstructive lung disease; hsCRP, high sensitive C-reactive protein; HGS, hand grip strength of dominant hand.

\section{Amino Acid-Based Profile Between Different BODEs}

In COPD patients from BODE 1 to BODE 4, there was a significant trend of increases in the levels of histidine, ornithine, phenylalanine, Hc, Oc, and Pc (Table 2). No significant changes were noted in leucine levels. Leucine levels increased insignificantly from BODE 1 to BODE 3 but decreased in BODE 4. Next, we investigated whether metabolites could discriminate patients with different BODE indexes. We merged patients with BODE 3 and 4 into one group due to severity and small sample size, and compared patients with BODE 3 and 4 to those with BODE 1. In univariate analysis, histidine, phenylalanine, $\mathrm{Hc}$ and $\mathrm{Pc}$ significantly discriminated patients with BODE indexes of 3 and 4 from those at BODE stage 1 (Table 4). In multivariable analysis, histidine and $\mathrm{Pc}$ were two independent variables useful for discrimination, and were consequently used to develop a "metabolic score". Based on the $\beta$ estimates from the multivariable logistic regression, the metabolic score was calculated as $0.049 \times$ histidine + $0.066 \times$ Pc. To discriminate patients at BODE stages 3 and
4 from those at BODE stage 1, the metabolic score had an OR of $2.74(95 \% \mathrm{CI}=1.41-5.29, \mathrm{p}=0.003)($ Table 5).

We also tested whether parameters of nutrition, inflammation and muscular strength were able to discriminate patients with BODE of 3 and 4 from those at BODE stage 1 (Table 5). Using univariate analysis, hand grip strength was the only significant parameter. Next, in multivariable analysis, we adjusted the metabolic score for hand grip strength. The value of the metabolic score for discriminating BODE 3 and 4 patients from BODE 1 patients was independent of hand grip strength (Table 5). Using ROC curves to evaluate the ability of discrimination, the metabolic score had an AUC of 0.773 (Figure 2). A combination of metabolic score and hand grip strength $(0.985 \times$ metabolic score $-0.094 \times$ hand grip strength +5 ) improved the AUC to 0.802 . Based on Youden's index, the cutoff values for the metabolic score and for a combination of metabolic score and hand grip strength were set at 9.53 and 9.66 , respectively. A metabolic score of $\geq 9.53$ significantly distinguished patients at BODE stages 3 and 4 from patients at BODE stage $1(\mathrm{OR}=15.75,95 \% \mathrm{CI}=3.60-68.88, \mathrm{p}<0.001)$ (the sensitivity, specificity, and accuracy were $63.6 \%, 90.0 \%$, 
Table 3 Univariate And Multivariable Analysis Of Metabolites In Discriminating Patients With Chronic Obstructive Lung Disease From Normal Controls

\begin{tabular}{|c|c|c|c|c|}
\hline \multirow[t]{2}{*}{ Amino Acid $(\mu \mathrm{M})$} & \multicolumn{2}{|l|}{ Univariate } & \multicolumn{2}{|l|}{ Multivariable* } \\
\hline & OR (95\% Cl) & p value & OR $(95 \% \mathrm{Cl})$ & p value \\
\hline Histidine & $0.98(0.96-1.01)$ & 0.160 & & \\
\hline Ornithine & $1.01(1.00-1.03)$ & 0.117 & & \\
\hline Leucine & $0.96(0.94-0.97)$ & $<0.001$ & $0.95(0.93-0.97)$ & $<0.001$ \\
\hline Phenylalanine & $0.98(0.95-1.01)$ & 0.118 & & \\
\hline $\mathrm{Hc}$ & $1.03(1.00-1.05)$ & 0.037 & & \\
\hline Oc & $1.03(1.01-1.04)$ & 0.001 & $1.04(1.02-1.06)$ & 0.001 \\
\hline $\mathrm{Pc}$ & $1.03(1.00-1.06)$ & 0.105 & & \\
\hline
\end{tabular}

Note: *Multivariable analysis with forward selection model for all variables.

Abbreviations: $\mathrm{OR}$, odds ratio; $\mathrm{Cl}$, confidence interval; $\mathrm{Hc}$, corrected histidine; Oc, corrected ornithine; Pc, corrected phenylalanine.

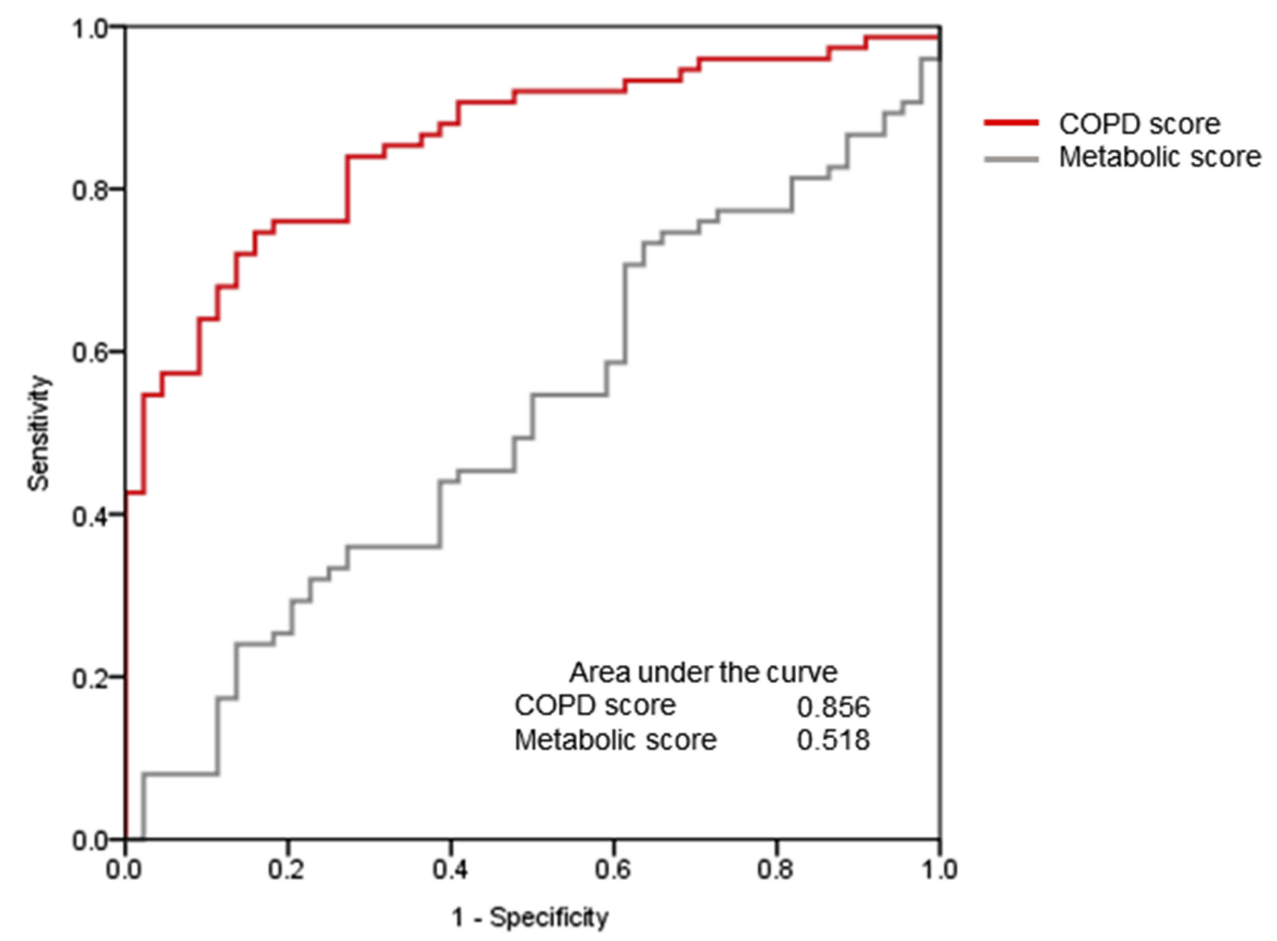

Figure I The receiver operating characteristic curves and the area under the curves of COPD score and metabolic score for discriminating patients with chronic obstructive pulmonary disease from normal controls. COPD, chronic obstructive pulmonary disease.

$77.3 \%$, respectively), and so did a combination of metabolic score and hand grip strength of $\geq 9.66$ (OR $=9.50,95 \%$ $\mathrm{CI}=2.30-39.30, \mathrm{p}=0.002)($ the sensitivity, specificity, and accuracy were $86.4 \%, 60.0 \%, 71.2 \%$, respectively). In addition, the metabolic score $\geq 9.53$ also significantly discriminated patients at BODE 3 and 4 from those at BODE 1 and $2(\mathrm{OR}=$ $8.56,95 \% \mathrm{CI}=2.77-26.39, \mathrm{p}<0.001)$.

\section{Discussion}

This study developed amino acid-based indexes, namely COPD score and metabolic score, to discriminate COPD patients from normal controls and to identify patients in advanced BODE stages from all COPD patients, respectively. COPD patients had lower leucine but higher ornithine concentrations compared to the normal controls. However, among patients with COPD, blood concentrations of histidine, ornithine and phenylalanine increased significantly along with disease severity from BODE stages 1 to 4 . The value of these scores in discrimination was better than parameters for nutrition, inflammation, and muscular function. In addition, the amino acid-based profile of COPD patients was different from that of patients with HF. 
Table 4 Univariate And Multivariable Analysis Of Metabolites In Discriminating Patients With BODE 3 And 4 From Patients With BODE I

\begin{tabular}{|c|c|c|c|c|}
\hline \multirow[t]{2}{*}{ Amino Acid $(\mu \mathrm{M})$} & \multicolumn{2}{|l|}{ Univariate } & \multicolumn{2}{|l|}{ Multivariable* } \\
\hline & OR (95\% Cl) & p value & OR $(95 \% \mathrm{Cl})$ & p value \\
\hline Histidine & $1.04(1.00-1.08)$ & 0.046 & $1.05(1.01-1.10)$ & 0.026 \\
\hline Ornithine & $1.02(1.00-1.04)$ & 0.082 & & \\
\hline Leucine & $1.01(0.98-1.03)$ & 0.605 & & \\
\hline Phenylalanine & $1.06(1.00-1.12)$ & 0.036 & & \\
\hline $\mathrm{Hc}$ & $1.04(1.01-1.08)$ & 0.027 & & \\
\hline Oc & $1.02(1.00-1.04)$ & 0.057 & & \\
\hline $\mathrm{Pc}$ & $1.06(1.01-1.11)$ & 0.018 & $1.07(1.02-1.13)$ & 0.011 \\
\hline
\end{tabular}

Note: *Multivariable analysis with forward selection model for all variables.

Abbreviations: OR, odds ratio; $\mathrm{Cl}$, confidence interval; $\mathrm{Hc}$, corrected histidine; Oc, corrected ornithine; Pc, corrected phenylalanine.

Table 5 Univariate And Multivariable Analysis Of The Metabolic Score And Clinical Parameters In Discriminating Patients With BODE 3 And 4 From Patients With BODE I

\begin{tabular}{|c|c|c|c|c|}
\hline & \multicolumn{2}{|l|}{ Univariate } & \multicolumn{2}{|l|}{ Multivariable* } \\
\hline & OR $(95 \% \mathrm{Cl})$ & $p$ value & OR $(95 \% \mathrm{CI})$ & $p$ value \\
\hline Age (years) & $1.03(0.96-1.10)$ & 0.467 & & \\
\hline Sex & $1.54(0.26-9.26)$ & 0.638 & & \\
\hline Metabolic score & $2.74(1.4 I-5.29)$ & 0.003 & $2.68(1.36-5.28)$ & 0.004 \\
\hline HGS (kg) & $0.91(0.85-0.99)$ & 0.021 & $0.91(0.83-1.00)$ & 0.040 \\
\hline $\mathrm{HsCRP}(\mathrm{mg} / \mathrm{L})$ & $1.06(0.98-1.15)$ & 0.122 & & \\
\hline Albumin $(g / d l)$ & $0.53(0.15-1.80)$ & 0.306 & & \\
\hline Pre-Albumin (mg/dL) & $0.93(0.85-1.02)$ & 0.121 & & \\
\hline Transferrin (mg/dL) & $0.99(0.97-1.00)$ & 0.107 & & \\
\hline
\end{tabular}

Note: *Multivariable analysis with forward selection model for all variables.

Abbreviations: OR, odds ratio; $\mathrm{Cl}$, confidence interval; $\mathrm{HGS}$, hand grip strength of dominant hand; hsCRP, high sensitive C-reactive protein.

Although we hoped the amino acid profile developed for HF could be applied for COPD, we found that the changes in these amino acids that accompanied with increasing severity of COPD were different from those in HF. In both diseases, ornithine and phenylalanine increased similarly with severity; however, the increase in histidine level in COPD contrasted to its decrease in HF. Moreover, even the differences in the amino acids between normal subjects and COPD were not similar to the findings in the HF population. The distinct COPD score could discriminate COPD patients from normal controls, independent of age and inflammation. This score helps the diagnosis of COPD patients who had poor performance of pulmonary function test or history taking, and definitely identifies a population with distinct metabolic discturbance associated with COPD in addition to lung function test-based diagnosis.

Muscular dysfunction is an important characteristic of COPD patients, which may be explained by decreased muscle synthesis and impaired muscle anabolism and hypertrophy due to decreased insulin like growth factor 1 , especially in cachexic COPD patients and during acute exacerbation. ${ }^{23,24}$ When histidine is used to synthesize muscle, it is turned into methylhistidine via methyltransferase enzyme during the cross-linking process of actin and myosine. ${ }^{9,15}$ Reduced utilization of histidine for muscle growth may result in increased serum histidine levels. Actually, previous studies have shown that serum histidine levels are elevated in COPD patients who had worse disease severity with emphysema, cachexia, and increased systemic inflammation. ${ }^{8,9}$ Interestingly, in HF patients, histidine level decreased with worse disease severity. One of the possible explanations was that HF is associated with greatly increased histidine consumption for energy production without adequately refilling the histidine pool, due to high turnover rate in muscle. ${ }^{15}$ The different amino acid profiles of COPD and HF patients require further investigations. 


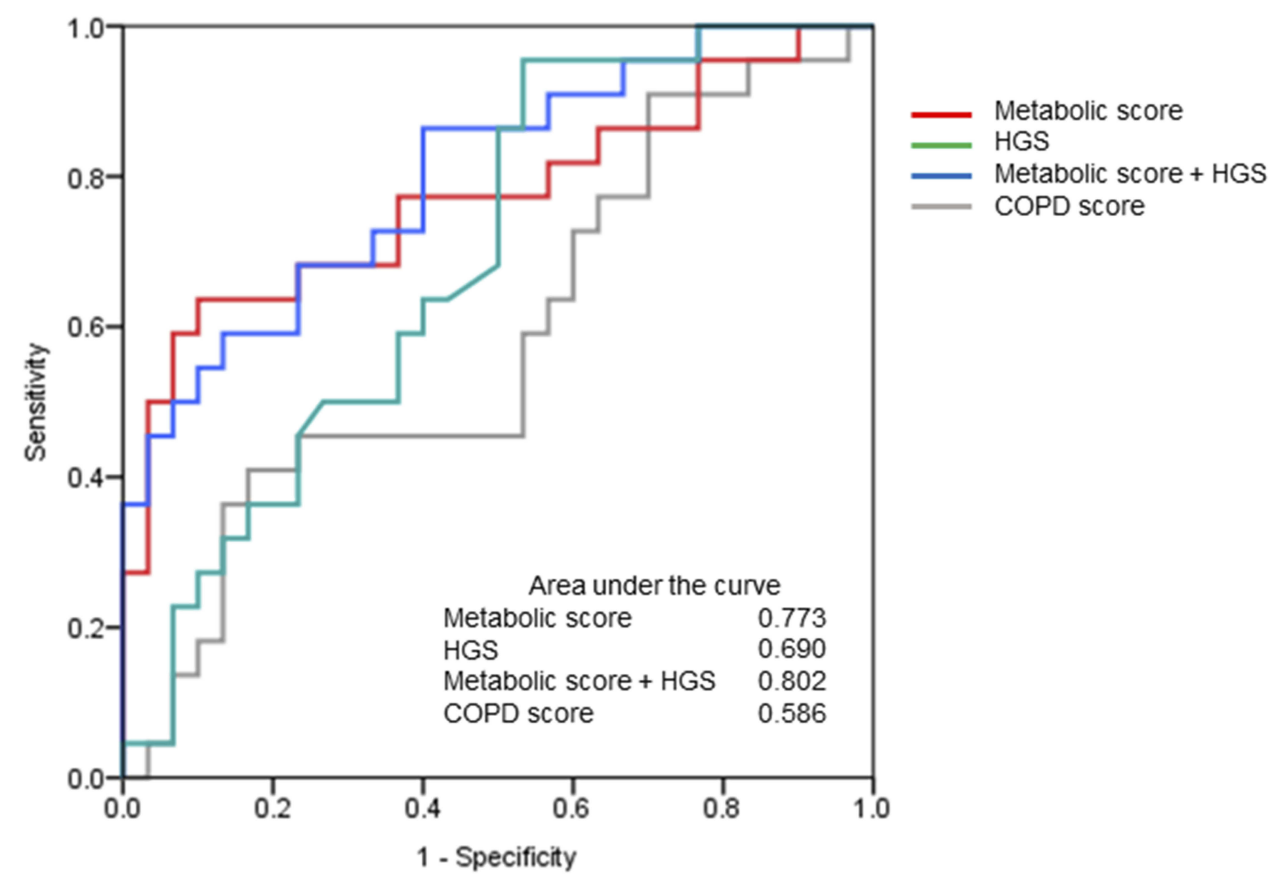

Figure 2 The receiver operating characteristic curves and the area under the curves of COPD score, metabolic score, hand grip strength (HGS), and a combination of metabolic score and HGS for discriminating patients at BODE 3/4 from patients at BODE I. COPD, chronic obstructive pulmonary disease.

COPD is characterized by lung inflammation due to inhaling noxious particles and increased number of macrophages, neutrophils and lymphocytes. ${ }^{25}$ Numerous inflammatory cytokines are then produced and released along with synthesis of inducible nitric oxide synthase by macrophages, epithelium, and vascular smooth muscle in airways. ${ }^{26}$ Previous studies have showed that the amount of nitric oxide in exhaled air was substantially increased among patients with more severe COPD and during acute exacerbations. $^{27-29}$ Nitric oxide inhibited ornithine decarboxylase. ${ }^{30,31}$ Previous studies have also demonstrated increased arginase activity in the airways of COPD patients, ${ }^{32}$ which indicates greater conversion of arginine to ornithine. ${ }^{33,34}$ Moreover, COPD is associated with mitochondrial dysfunction, ${ }^{24}$ which potentially causes dysregulation of the urea cycle, followed by increased ornithine metabolism. These sophisticated mechanisms may contribute to the elevated ornithine levels from BODE stages 1 to 4 , as noted in our study.

In cachexia, protein breakdown driven by cytokines or metabolized for fuel production releases amino acids. ${ }^{35}$ Phenylalanine, which serves as both a glucogenic and ketogenic amino acid, degrades to enter the Krebs cycle or is converted to acetoacetyl-CoA to form ketone bodies. ${ }^{35}$ In COPD patients with cachexia, phenylalanine is elevated, along with histidine, glutamine, arginine, and aspartic acid, compared with non-cachexia patients. ${ }^{9}$ Moreover, increased net release of phenylalanine in skeletal muscle is observed when fasting, which is related to reduced mTOR activation. ${ }^{36}$ The elevated phenylalanine concentrations in COPD may be associated with increased muscle breakdown due to cachexia. Furthermore, chronic inflammation and exaggerated oxidative stress, which are characteristics of COPD, substantially lower the bioavailability of 5,6,7,8tetrahydrobiopterin which is a co-factor for conversion of phenylalanine to tyrosine. This mechanism also potentially increases phenylalanine levels. ${ }^{37}$

As demonstrated in our study, the metabolic score is able to identify COPD patients at BODE stages 3 and 4 . However, its combination with other clinical parameters merits further consideration for clinical application. For example, hand grip strength and metabolic score represented independent aspects of COPD assessment, and their combination may objectively improve identification of patients at high risk, especially for those who cannot walk.

A number of confounding factors may interfere with the interpretation of metabolism, including age, nutritional status, muscle mass, and more. In our study, the normal controls were younger than our COPD patients. Previously, we showed that leucine was strongly correlated with the total amount of essential amino acids ${ }^{12}$ which indicates nutritional status and decreases with aging. ${ }^{38}$ Thus, to overcome 
the effect of differences in patient characteristics on metabolite concentrations, we used leucine to correct histidine, ornithine and phenylalanine levels. Based on corrections we proposed in the Methods section, there were no significant differences in the histidine and phenylalanine levels between the normal controls and BODE stage 1 patients. On the other hand, the decreased leucine levels compared to normal subjects also warrant further investigations on the associated mechanisms.

The study has several limitations. First, the mean age of our COPD patients was over 70 , which reflects the natural course of COPD. However, it was difficult to recruit healthy individuals over age 70 without any comorbidities for the control group. This made fair evaluation of the differences in metabolites between COPD patients and healthy controls difficult. Second, the metabolic score should be compared head to head with BODE index based on the outcomes, such as mortality. However, the follow-up duration was not long enough in our study population. Third, although the LVEFs were normal (average $\mathrm{LVEF}=66.7 \pm 9.0 \%$ ) in our patients, cardiac echocardiograms were not performed in $15 \%$ of COPD patients. However, these patients had no symptoms, signs, or evidence of HF when COPD was not exacerbated. Fourth, since the sample size in this study was small, studies with a large sample size are indispensable for ensuring the value of the COPD and metabolic scores. Finally, this study tested the value of a successful amino acid panel derived from $\mathrm{HF}$ patients for patients with COPD. To create a customized metabolic profile for COPD patients, we recommend untargeted metabolomics-based mining of plasma metabolites for future research.

\section{Conclusions}

Based on a simple amino acid profile, the COPD score provides a metabolic signature of COPD independent of age and inflammatory status, and helps diagnosis of COPD. The metabolic score is capable of objectively identifying patients with severe forms of COPD. These finding raise the possibility of using biomarkers to replace the BODE index, which cannot be used for patients who are unable to walk or provide accurate information on the components of BODE. Future studies will further explore the characteristics of patients with high COPD scores, and focus on whether a metabolic score provides prognostic value similar to or better than BODE.

\section{Abbreviations}

COPD, Chronic obstructive lung disease; BMI, body mass index; FEV1, forced expiratory volume in one second;
mMRC, modified Medical Research Council questionnaire; 6MWD, six-minute walking distance; HF, heart failure; UPLC, ultra-performance liquid chromatography; CAT, chronic obstructive lung disease assessment test; $\mathrm{SpO}$, oxygen saturation; hsCRP, high sensitive C-reactive protein; Hc, corrected histidine level; Oc, corrected ornithine levels; Pc, corrected phenylalanine levels; ROC, receiver operating characteristic; AUCs, area under curves.

\section{Acknowledgments}

This work was supported by Chang Gung Memorial Hospital (CMRPG2G0621). We thank Yi-Tsen Lin for providing nutritional evaluation support of our study population. We also thank the Healthy Aging Research Center, Chang Gung University and the Featured Areas Research Center Program within the Framework of the Higher Education Sprout Project by the Ministry of Education in Taiwan.

\section{Disclosure}

The authors report no conflicts of interest in this work.

\section{References}

1. López-Campos JL, Tan W, Soriano JB. Global burden of COPD. Respirology. 2016;21(1):14-23. doi:10.1111/resp.12660

2. Mirza S, Clay RD, Koslow MA, Scanlon PD COPD guidelines: a review of the 2018 GOLD report. Paper presented at: Mayo Clinic Proceedings 2018.

3. Celli BR, Cote CG, Marin JM, et al. The body-mass index, airflow obstruction, dyspnea, and exercise capacity index in chronic obstructive pulmonary disease. $N$ Engl J Med. 2004;350(10):1005-1012. doi:10.1056/NEJMoa021322

4. Ong K-C, Earnest A, Lu S-J. A multidimensional grading system (BODE index) as predictor of hospitalization for COPD. Chest. 2005;128(6):3810-3816. doi:10.1378/chest.128.6.3810

5. Marin JM, Carrizo SJ, Casanova C, et al. Prediction of risk of COPD exacerbations by the BODE index. Respir Med. 2009;103(3):373378. doi:10.1016/j.rmed.2008.10.004

6. Jones P, Agusti A. Outcomes and markers in the assessment of chronic obstructive pulmonary disease. Eur Respir J. 2006;27 (4):822-832. doi:10.1183/09031936.06.00145104

7. Beger RD, Dunn W, Schmidt MA, et al. Metabolomics enables precision medicine:"a white paper, community perspective". Metabolomics. 2016;12(9):149. doi:10.1007/s11306-016-1094-6

8. Ubhi BK, Riley JH, Shaw PA, et al. Metabolic profiling detects biomarkers of protein degradation in COPD patients. Eur Respir J. 2012;40(2):345-355. doi:10.1183/09031936.00112411

9. Ubhi BK, Cheng KK, Dong J, et al. Targeted metabolomics identifies perturbations in amino acid metabolism that sub-classify patients with COPD. Mol Biosyst. 2012;8(12):3125-3133. doi:10.1039/ $\mathrm{c} 2 \mathrm{mb} 25194 \mathrm{a}$

10. Chen Q, Deeb RS, Ma Y, Staudt MR, Crystal RG, Gross SS. Serum metabolite biomarkers discriminate healthy smokers from COPD smokers. PLoS One. 2015;10(12):e0143937.

11. Bregy L, Nussbaumer-Ochsner Y, Sinues PM-L, et al. Real-time mass spectrometric identification of metabolites characteristic of chronic obstructive pulmonary disease in exhaled breath. Clin Mass Spectrom. 2018;7:29-35. 
12. Wang C-H, Cheng M-L, Liu M-H. Simplified plasma essential amino acid-based profiling provides metabolic information and prognostic value additive to traditional risk factors in heart failure. Amino Acids. 2018;50(12):1739-1748.

13. Snowden S, Dahlén S-E, Wheelock CE. Application of metabolomics approaches to the study of respiratory diseases. Bioanalysis. 2012;4(18):2265-2290. doi:10.4155/bio.12.218

14. Cheng M-L, Wang C-H, Shiao M-S, et al. Metabolic disturbances identified in plasma are associated with outcomes in patients with heart failure: diagnostic and prognostic value of metabolomics. $J \mathrm{Am}$ Coll Cardiol. 2015;65(15):1509-1520. doi:10.1016/j.jacc.2015. 02.018

15. Wang C-H, Cheng M-L, Liu M-H. Amino acid-based metabolic panel provides robust prognostic value additive to b-natriuretic peptide and traditional risk factors in heart failure. Dis Markers. 2018;2018.

16. de Miguel Díez J, Morgan JC, García RJ. The association between COPD and heart failure risk: a review. Int J Chron Obstruct Pulmon Dis. 2013;8:305. doi:10.2147/COPD.S31236

17. Anker SD, Sharma R. The syndrome of cardiac cachexia. Int $J$ Cardiol. 2002;85(1):51-66. doi:10.1016/s0167-5273(02)00233-4

18. Dempsey TM, Scanlon PD Pulmonary function tests for the generalist: a brief review. Paper presented at: Mayo Clinic Proceedings 2018.

19. Bestall J, Paul E, Garrod R, Garnham R, Jones P, Wedzicha J. Usefulness of the Medical Research Council (MRC) dyspnoea scale as a measure of disability in patients with chronic obstructive pulmonary disease. Thorax. 1999;54(7):581-586. doi:10.1136/thx.54.7. 581

20. Jones P, Harding G, Berry P, Wiklund I, Chen W, Leidy NK. Development and first validation of the COPD assessment test. Eur Respir J. 2009;34(3):648-654. doi:10.1183/09031936.00102509

21. Laboratories ACoPSfCPF. ATS statement: guidelines for the sixminute walk test. Am J Respir Crit Care Med. 2002;166:111-117. doi:10.1164/ajrccm.166.1.at1102

22. Rantanen T, Masaki K, Foley D, Izmirlian G, White L, Guralnik J. Grip strength changes over 27 yr in Japanese-American men. $J$ Appl Physiol. 1998;85(6):2047-2053. doi:10.1152/jappl.1998.85.6.2047

23. Gea J, Agustí A, Roca J. Pathophysiology of muscle dysfunction in COPD. J Appl Physiol. 2013;114(9):1222-1234. doi:10.1152/ japplphysiol.00981.2012

24. Remels A, Gosker HR, Langen RC, Schols AM. The mechanisms of cachexia underlying muscle dysfunction in COPD. J Appl Physiol. 2012;114(9):1253-1262. doi:10.1152/japplphysiol.00790.2012

25. Pesci A, Balbi B, Majori M, et al. Inflammatory cells and mediators in bronchial lavage of patients with chronic obstructive pulmonary disease. Eur Respir J. 1998;12(2):380-386. doi:10.1183/09031936. 98.12020380
26. Hogg JC, Chu F, Utokaparch S, et al. The nature of small-airway obstruction in chronic obstructive pulmonary disease. $N$ Engl $J$ Med. 2004;350(26):2645-2653. doi:10.1056/NEJMoa032158

27. Ansarin K, Chatkin J, Ferreira I, Gutierrez C, Zamel N, Chapman K. Exhaled nitric oxide in chronic obstructive pulmonary disease: relationship to pulmonary function. Eur Respir J. 2001;17(5):934-938. doi: 10.1183/09031936.01.17509340

28. Brindicci C, Ito K, Resta O, Pride N, Barnes P, Kharitonov S. Exhaled nitric oxide from lung periphery is increased in COPD. Eur Respir J. 2005;26(1):52-59. doi:10.1183/09031936 .04 .00125304

29. Lu Z, Huang W, Wang L, Xu N, Ding Q, Cao C. Exhaled nitric oxide in patients with chronic obstructive pulmonary disease: a systematic review and meta-analysis. Int $J$ Chron Obstruct Pulmon Dis. 2018;13:2695-2705. doi:10.2147/COPD.S165780

30. Hu J, Mahmoud MI, El-Fakahany EE. Polyamines inhibit nitric oxide synthase in rat cerebellum. Neurosci Lett. 1994;175(1-2):41-45. doi:10.1016/0304-3940(94)91073-1

31. Bauer PM, Fukuto JM, Buga GM, Pegg AE, Ignarro LJ. Nitric oxide inhibits ornithine decarboxylase by S-nitrosylation. Biochem Biophys Res Commun. 1999;262(2):355-358. doi:10.1006/bbrc.1999.1210

32. J-M T, Henno P, Leroy I, et al. Role of the nitric oxide synthase/ arginase balance on bronchial reactivity in patients with chronic obstructive pulmonary disease. Am J Physiol Lung Cell Mol Physiol. 2008;294:L489-L497.

33. Maarsingh H, Pera T, Meurs H. Arginase and pulmonary diseases. Naunyn Schmiedebergs Arch Pharmacol. 2008;378(2):171. doi:10.1007/s00210-008-0286-7

34. Pera T, Zuidhof A, Smit M, et al. Arginase inhibition prevents inflammation and remodeling in a guinea pig model of chronic obstructive pulmonary disease. J Pharmacol Exp Ther. 2014;349 (2):229-238. doi:10.1124/jpet.113.210138

35. Murray AJ, Montgomery HE. How wasting is saving: weight loss at altitude might result from an evolutionary adaptation. Bioessays. 2014;36(8):721-729. doi:10.1002/bies.201400042

36. Vendelbo MH, Møller AB, Christensen B, et al. Fasting increases human skeletal muscle net phenylalanine release and this is associated with decreased mTOR signaling. PLoS One. 2014;9(7): e102031.

37. Neurauter G, Schrocksnadel K, Scholl-Burgi S, et al. Chronic immune stimulation correlates with reduced phenylalanine turnover. Curr Drug Metab. 2008;9(7):622-627.

38. Pitkänen H, Oja S, Kemppainen K, Seppä J, Mero A. Serum amino acid concentrations in aging men and women. Amino Acids. 2003;24 (4):413-421. doi:10.1007/s00726-002-0338-0

\section{Publish your work in this journal}

The International Journal of COPD is an international, peer-reviewed journal of therapeutics and pharmacology focusing on concise rapid reporting of clinical studies and reviews in COPD. Special focus is given to the pathophysiological processes underlying the disease, intervention programs, patient focused education, and self management protocols. This journal is indexed on PubMed Central, MedLine and CAS. The manuscript management system is completely online and includes a very quick and fair peer-review system, which is all easy to use. Visit http://www.dovepress.com/testimonials.php to read real quotes from published authors. 